\title{
MAPPING OF STRUCTURAL GENES FOR THE ENZYMES OF CYSTEINE BIOSYNTHESIS IN ESCHERICHIA COLI K12 AND SALMONELLA TYPHIMURIUM LT2
}

\author{
M. C. JONES-MORTIMER* \\ Department of Genetics, University of Birmingham, P.O. Box 363, Birmingham B15 2 TT
}

Received 1.x.72

\section{SUMmary}

Deletion analysis of the episome from strain CB075 shows that the order and orientation of the structural genes for cysteine biosynthesis in Escherichia coli is

\section{lysA thy A argA cysfIHDC}

In Salmonella typhimurium the orientation of the corresponding genes was shown by a four-point conjugation cross to be

$$
\text { thy } A \arg B \text { cys } C(D H I) \mathcal{F}
$$

Despite this inversion the structure of the gene clusters may be similar. If so, this is relevant to the study of the evolution of enteric bacteria. Complementation tests showed that $E$. coli gene formerly called cys $P$ is functionally equivalent to the $S$. typhimurium gene cys $\mathcal{F}$.

\section{INTRODUCTION}

Demerec and Ohta (1964) showed that the homology between Escherichia coli K12 and Salmonella typhimurium LT2 is particularly low in the cysC region. Preliminary results from this laboratory (cited by Smith, 1971), suggested that the order of the cys structural genes $C D H I F$ in the two organisms might not be identical. To resolve this problem a deletion map of the cys $C D H I F$ genes of $E$. coli was constructed, by a modification of the technique of Marsh and Duggan (1972). Whereas these authors used a series of episomes shortened by transduction, this paper describes the shortening of episomes by spontaneous mutation. Which genes remain on the shortened episomes can be determined by complementation, and since shortening is, in each case, the result of a single event the resulting complementation map is equivalent to a deletion map of the same region.

The experiments here described show that the order of the cysCDHIf genes is the same in the two organisms, and suggest that the structure of the group may be similar, though its orientation is different.

\section{Materials AND METHODS}

(i) Media

The minimal medium was that of Smith (1961) supplemented with thymine $(60 \mu \mathrm{g} . / \mathrm{ml}$.), L-lysine $(80 \mu \mathrm{g} . / \mathrm{ml}$.$) , L-arginine (40 \mu \mathrm{g} . / \mathrm{ml}$.$) other$ L-amino acids $(20 \mu \mathrm{g} . / \mathrm{ml}$.) trimethoprim $(5 \mu \mathrm{g} . / \mathrm{ml}$.), and thiamin $(2 \mu \mathrm{g} . / \mathrm{ml})$. as required. Glucose or lactose $(0.4$ per cent) was used as the carbon source.

Oxoid nutrient broth, Oxoid nutrient agar and EMB lactose agar were

* Present address: Department of Biochemistry, University of Leicester. 
routinely supplemented with thymine and cystine at the same concentrations as used for minimal medium.

All incubations were at $37^{\circ} \mathrm{C}$.

(ii) Bacteria

The strains listed in tables $1 a$ and $1 b$ were employed. All Cys ${ }^{-}$strains were checked for their growth response to $1 \mathrm{mM}$ cysteine sulphinic acid:

\begin{tabular}{|c|c|c|}
\hline \multicolumn{3}{|c|}{ TABLe $1 a$} \\
\hline & E. coli strains & Origin \\
\hline 5K-C600 & $\mathrm{F}-$ thr leu thi lac $\mathrm{r}^{-} \mathrm{m}_{\mathrm{K}}^{+}$ & S. Glover \\
\hline JM57 & F-cysI thi & $\mathrm{AB} 1621$ \\
\hline *JM73 & F-cysf $(P)$ thr leu trp his arg thi & PA309 \\
\hline JM81 & $\mathrm{F}-\mathrm{cys} C$ & *JM41 \\
\hline *JM96 & F-cysH thr leu trp his arg thi & PA309 \\
\hline JM221 & $\mathrm{F}-c y s D$ pro his $i l v A$ arg $A$ trp & JM201 \\
\hline JM245 & $\begin{array}{l}\text { his argE leu metB thy lac y strA recAI } \\
\mathrm{F}^{\prime} \text { lac }{ }^{+} \text {lys } A^{+} \text {thy } A^{+} \text {arg } A^{+} \text {cys } \mathcal{F} I H D C^{+}\end{array}$ & $\begin{array}{l}\mathrm{F}^{\prime} \text { from } \mathrm{CB} 075 \\
\text { in JC1553 thy }\end{array}$ \\
\hline
\end{tabular}

* These strains have been described (Jones-Mortimer, 1968).

Table $1 b$

\begin{tabular}{|c|c|c|}
\hline \multirow{2}{*}{\multicolumn{2}{|c|}{ S. typhimurium strains }} & \multirow[b]{2}{*}{ Origin } \\
\hline & & \\
\hline 0015 & $\operatorname{argB0060}$ cys $C 0849$ & \\
\hline 0019 & lys $A 0080$ & \\
\hline 0078 & HfrB3 cysCO200 hisD 0023 & \\
\hline 0236 & cysCD 519 & \\
\hline 0240 & cysC537 & Dirmingnamin cuture conection \\
\hline 0251 & cysH75 & \\
\hline 0259 & cysI68 & \\
\hline 0266 & cys 7538 & \\
\hline JM263 & cysC thy & 0240 \\
\hline JM265 & cys $H$ thy & 0251 \\
\hline JM266 & cysI thy & 0259 \\
\hline JM267 & cysf thy & 0266 \\
\hline JM268 & cys $C$ thy $\mathrm{F}^{\prime}$ cys $C D H 1 \mathcal{F}^{+}$thy+ lac $^{+}$ & JM263×JM245 \\
\hline JM270 & cys $H$ thy $\mathrm{F}^{\prime}$ cys $C D H \mathrm{~F}^{+}$thy+ $\mathrm{lac}^{+}$ & $\mathrm{JM} 265 \times \mathrm{JM} 245$ \\
\hline JM271 & cysI thy $\mathrm{F}^{\prime}$ cys $C D H I \mathcal{F}^{+}$thy+ lac ${ }^{+}$ & JM266 × JM245 \\
\hline JM272 & cysf thy $\mathrm{F}^{\prime}$ cys $\mathrm{CDHIF} \mathrm{f}^{+}$thy+ $\mathrm{lac}^{+}$ & JM267 ×JM245 \\
\hline JM278 & HfrB3 $c y s C$ hisD argB & $\begin{array}{l}\text { transduction of a thy } A \text { mutant of } \\
0078 \text { with } 0015 \text { as donor }\end{array}$ \\
\hline JM279 & $\operatorname{cys} f \arg B$ & $\begin{array}{l}\text { transduction of JM267 with } 0015 \\
\text { as donor }\end{array}$ \\
\hline JM280 & cysf argB thy & JM279 \\
\hline JM292 & cysf argB thy $\mathrm{F}^{\prime}$ cysCDHIf+ arg+ thy+ lac ${ }^{+}$ & $\mathrm{JM} 280 \times \mathrm{JM} 245$ \\
\hline JM321 & cys $H$ thy $\mathrm{F}^{\prime}$ cysCDH $H^{+}$ & \\
\hline JM323 & cysh thy $\mathrm{F}^{\prime}$ cys $C D^{+}$ & see text and table 2 \\
\hline JM329 & cysC thy $\mathrm{F}^{\prime}$ cys $C^{+}$ & \\
\hline JM353 & cysI thy $\mathrm{F}^{\prime}$ cys $C D H I^{+}$ & \\
\hline
\end{tabular}

cys $C, D$ and $H$ mutants grew, cys $I$ and $\mathcal{J}$ mutants did not. Furthermore all strains used showed self-consistent complementation patterns. (Smith (1971) has reviewed the biochemistry of these mutants.)

The nomenclature of Demerec, Adelberg, Clark and Hartman (1966) is employed. The reader is warned that the symbols $\arg A(E$. coli) and $\arg B$ (S. typhimurium) represent homologous genes. 


\section{(iii) Bacteriophage}

P22 int 4 was obtained from the Birmingham stock collection.

\section{(iv) Selection of thymineless strains}

Spontaneous thymineless derivatives of both haploids and diploids were obtained by plating 1-5 drop aliquots of the parental strains (grown overnight from single colonies in $2 \mathrm{ml}$. nutrient broth) on minimal medium supplemented with thymine and trimethoprim (Stacey and Simson, 1965) and other supplements as required. The plates were incubated 24-36 hours. The Thy mutant of 0078 was induced by a crystal of N-methyl N-nitroso $N^{\prime}$-nitroguanidine placed on the selection medium.

\section{(v) Transfer of episome to $S$. typhimurium}

Strain JM245 was mated with strains of $S$. typhimurium (Hayes, 1957) for 1 hour and $0.1 \mathrm{ml}$. aliquots plated on lactose minimal agar, supplemented with any compounds required by the recipient. The plates were incubated for 24 hours. Single colony isolates were made on EMB lactose, and shown to be $\mathrm{Lac}^{+}$prototrophs but to segregate $\mathrm{Lac}^{-}$auxotrophs of the expected phenotype on restreaking.

\section{(vi) Transduction}

The transducing phage was propagated by the method of Swanstrom and Adams (1951). Transduction was carried out by spreading 1 drop of the phage lysate and 3 drops of an overnight culture of the recipient on suitable selective medium. Recombinants were screened by replica plating and single colony isolates made from one of the required type.

\section{(vii) Isolation of partially deleted episomes}

Merodiploids heterozygous for thy $A$ may become Thy- (i) by haploidisation, (ii) by mutation of the thy $A^{+}$allele, or (iii) by deletion of the thy $A^{+}$allele and neighbouring genes. Since the episome carries thy $A^{+}$and cysCDHIJ+ some deletions of thy $A^{+}$may also delete some but not all of the cys genes. A search was therefore made for strains with such deletions.

Spontaneous thymineless derivatives of strains JM270, JM271 and JM272 were selected on lactose minimal agar supplemented with cystine, thymine and trimethoprim. Since these colonies utilise lactose as the carbon source they cannot have arisen by mechanism (i). If they arose by mechanism (ii) they will grow on medium not supplemented with cystine. Colonies on the selection plates were therefore replica plated on to lactose minimal agar supplemented with thymine and trimethoprim and incubated for 24-30 hours. The majority of them failed to grow on this medium, indicating that an episomal cys $\mathrm{s}^{+}$function had been lost from these clones. It therefore is plausible that they arose by mechanism (iii). If so, some of the Cys- clones may still be capable of transmitting one or more of the episomal cyst genes. The Thy colonies were therefore also replica plated on to a lawn of strain JM263 (cysC thy) on lactose minimal agar supplemented with thymine and trimethoprim and incubated for 24-30 hours. Some clones (about 1 from $10^{8}$ cells plated) which did not grow on the first replica plate gave rise to colonies on the lawn, presumably because they were able to transmit cys $C^{+}$ and $\mathrm{lac}^{+}$to strain JM263. Such clones were picked (either off the master 
plate or, more usually, off the plate with the lawn) and streaked out on EMB lactose to obtain single colony isolates.

Trimethoprim was included in the replica plates to prevent the growth of 'Thy ${ }^{+}$cells: hence a thy cys strain had to be used for the lawn. Preliminary experiments with strains JM267 and JM268 were unsuccessful, for reasons which will subsequently prove obvious. Strain JM353 was obtained from strain JM272 by replica plating on to a lawn of strain JM266 (cysI thy).

\section{(viii) Plate mating}

Overnight cultures of the strains obtained were streaked on lawns of strains 0240 (cys $C), 0236$ (cysCD), 0251 (cysH), 0259 (cysI) and 0266 (cysf) on lactose minimal agar. The plates were incubated, and scored after 24 and 48 hours.

\section{(ix) Transfer of episomes to $E$. coli}

Overnight cultures of strains JM321, JM323, JM329 and JM353 were streaked on lawns of strain 5K-C600 (thr leu thi lac $\mathrm{r}^{-} \mathrm{m}_{\mathrm{K}}^{+}$) on lactose minimal medium supplemented with threonine, leucine and thiamin. The plates were incubated for about 36 hours. Colonies growing on them were picked and streaked on lawns of JC1553 thy on lactose minimal agar supplemented with leucine, methionine, arginine, histidine and thymine. These plates were incubated for 48 hours, and single colony isolates made from colonies growing on them. Overnight nutrient broth cultures of these isolates were tested for their ability to complement an $E$. coli cys $C$ mutant.

\section{REsults AND DIscussion}

(i) Order and orientation of the cysCDHIJ genes in $E$. coli

The selection procedure yielded strains all of which can transmit cys $C^{+}$ but not some or all of the other genes of this group. A complementation

TABle 2

Complementation pattern of derivative $F^{\prime}$ strains with $S$. typhimurium cys mutants

\begin{tabular}{|c|c|c|c|c|c|c|c|c|c|}
\hline \multirow[b]{2}{*}{$F^{\prime}$ type } & \multirow[b]{2}{*}{$\begin{array}{c}\text { Number } \\
\text { tested }\end{array}$} & \multirow[b]{2}{*}{$\begin{array}{l}\text { Donor } \\
\text { strain }\end{array}$} & \multirow[b]{2}{*}{$\begin{array}{l}\mathrm{F}^{\prime} \text { isolated } \\
\text { from }\end{array}$} & \multirow[b]{2}{*}{$F^{\prime}$ now in } & \multicolumn{5}{|c|}{ Recipient } \\
\hline & & & & & $\begin{array}{l}0240 \\
\text { cys } C\end{array}$ & $\begin{array}{r}0236 \\
\text { cysCD }\end{array}$ & $\begin{array}{l}0251 \\
\text { cys } H\end{array}$ & $\begin{array}{c}0259 \\
\text { cysI }\end{array}$ & $\begin{array}{l}0266 \\
\text { cys7 }\end{array}$ \\
\hline 1 & 1 & JM329 & JM272 & JM263 & + & - & - & - & - \\
\hline 2 & 12 & JM323 & JM270 & JM265 & + & + & - & - & - \\
\hline 3 & 6 & JM321 & JM270 & JM265 & + & + & + & - & - \\
\hline 4 & 0 & JM353 & $\mathrm{JM} 272$ & JM266 & + & + & + & + & - \\
\hline
\end{tabular}

+ confluent growth after 24 hours.

- no confluent growth after 44 hours (in some cases a few discrete colonies were obtained).

* This column gives the numbers of strains of each type selected in strain JM272 by backcrossing to strain JM263.

map can thus be established. About 30 different variant donor strains were isolated, and found to complement with cys $C, D, H, I$ and $\mathcal{J}$ mutants as shown in table 2. This pattern of complementation is what is expected if the Thy- Lac ${ }^{+} \mathrm{Cys}^{-}$phenotype arises by loss of part of the episome. If so, the 
complementation map is equivalent to a deletion map, and the order of the genes must be

\section{thy $A$ cysf cys cys $H$ cys $D$ cys $C$}

Column 2 of table 2 shows the numbers of partially deleted episomes obtained from strain JM272 by crossing to strain JM263. The numbers in each class are clearly not equal $\left(\chi^{2}\right.$ for 3 degrees of freedom $=16$, $\mathrm{P}<0.001$ ). Such a result is expected either if the ends of deletions are randomly distributed and the intervals defined by the complementation map are unequal, or if the ends of deletions occur more readily in certain places. Whichever of these explanations is correct (they are not mutually exclusive), there is a parallel in S. typhimurium. In this organism there are silent sections between cys $I$ and $c y s H$ and between $c y s H$ and $c y s D$ (Demerec, Gillespie and Mizobuchi, 1963). If such silent sections exist also in $E$. coli rather than being artifacts of recombination in $S$. typhimurium they could account for the preponderance of episomes of type 2. On the other hand, Itikawa and Demerec (1967) showed that the most frequent class of cys deletion mutant in S. typhimurium, the so-called ditto deletions, end in a small region of the silent section between cys $D$ and cys $H$. The deletions of type 2 episomes also end between $c y s D$ and cysH (or within cys $H$ ).

\section{(ii) Validation of the $E$. coli mapping procedure}

It has been assumed so far that those clones selected as Thy' which are also Cys- became so by a single event leading to a deletion of part of the episome. This hypothesis predicts that none of the variant episomes isolated should carry $\arg A^{+}$(and therefore that none of them should complement with a $S$. typhimurium $\arg B$ mutant) if $\arg A$ lies between $\operatorname{thy} A$ and cys FIHDC. Twenty-two of the variant $\mathrm{F}^{\prime}$ strains were tested, but none complemented $\arg B$ (though the original thy ${ }^{+}$episome did so). Some complemented $l y s A$; others did not.

The hypothesis was further tested by the following experiment. Samples $\left(6.8 \times 10^{7}\right.$ cells) from overnight broth-grown cultures of strain JM292, (lac thy $A \arg B$ cys $f \mathrm{~F}^{\prime}$ lac ${ }^{+}$thy $A^{+} \arg A^{+}$cys $\mathcal{F}^{+}$) were plated either on glucose minimal agar supplemented with thymine, trimethoprim and arginine or on lactose minimal agar supplemented with thymine, trimethoprim, arginine and cystine. The plates were incubated for 44 hours and replicas then made, in the first case on to

(a) lactose minimal agar + thymine + trimethoprim

(b) glucose minimal agar + thymine + trimethoprim

(c) lactose minimal agar + thymine + trimethoprim + arginine

(d) glucose minimal agar + thymine + trimethoprim + arginine

and in the second case on to

(a) lactose minimal agar + thymine + trimethoprim

(b) lactose minimal agar + thymine + trimethoprim + cystine

(c) lactose minimal agar + thymine + trimethoprim + arginine

(d) lactose minimal agar + thymine + trimethoprim + cystine + arginine

The replica plates were incubated for 24 hours and the number of colonies on each scored. From these data the numbers of colonies of the different possible phenotypes were calculated. The results are given in table 3 . These 
figures were tested using a $2 \times 2$ contingency $\chi^{2}$ test to determine whether the variant colonies of phenotype $\mathrm{Lac}^{-} \mathrm{Arg}^{-}$or $\mathrm{Arg}^{-} \mathrm{Cys}^{-}$could have arisen by two independent events. The values of $\chi^{2}$ obtained were extremely large, indicating that such variants do not arise by independent events. It is therefore plausible that such variants arise by single deletions of part of the episome. The failure to detect any variants of phenotype $\mathrm{Thy}^{-} \mathrm{Arg}^{+} \mathrm{Cys}^{-}$ is highly significant and is compatible with the gene order thy $A \arg A \operatorname{cys} C$

TABLE 3

Thymineless variants of strain 7 M292

lac thy $A$ argB cysf $\quad \mathrm{F}^{\prime}$ lac ${ }^{+}$thy $A^{+}$arg $A^{+}$cys $\mathcal{f}^{+}$

Colonies selected as

\begin{tabular}{|c|c|c|c|c|c|c|c|c|c|}
\hline \multirow[b]{3}{*}{ of phenotype } & \multirow{5}{*}{$\begin{array}{l}\text { Cys } \\
\text { Arg } \\
\text { Lac }\end{array}$} & \multicolumn{8}{|c|}{ Colonies selected as } \\
\hline & & \multicolumn{4}{|c|}{ Thy- Lac ${ }^{+}$} & \multicolumn{4}{|c|}{ Thy- Cys ${ }^{+}$} \\
\hline & & + & + & - & - & & & & \\
\hline & & + & - & + & - & + & + & - & - \\
\hline & & 194 & 58 & 0 & c. 2000 & $\stackrel{+}{244}$ & $\overline{326}$ & $\stackrel{+}{160}$ & $\overline{464}$ \\
\hline \multicolumn{2}{|c|}{$2 \times 2$ contingency $\chi^{2}$} & \multicolumn{4}{|c|}{1685} & \multicolumn{4}{|c|}{$39 \cdot 2$} \\
\hline
\end{tabular}

(Taylor, 1970). The experiment shows the order of the genes carried on the episome to be

$$
\mathrm{F} l a c^{+} \operatorname{thy} A^{+} \arg A^{+} \operatorname{cys} \mathrm{f}^{+}
$$

as is predicted by its derivation from the transposition Hfr TOR 13 (Berg and Curtiss, 1967).

What is perhaps surprising about this experiment is that large deletions occur more frequently than small ones (or point mutations of thy $A$ ). There appear also to be more $\mathrm{Lac}^{+} \mathrm{Thy}^{-} \mathrm{Arg}^{-} \mathrm{Cys}^{-}$derivatives than $\mathrm{Cys}^{+} \mathrm{Thy}^{-}$

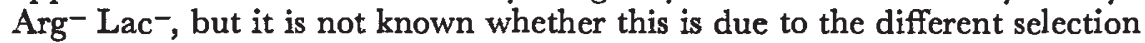
systems used or to some orientated effect in the establishment of deletions.

(iii) E. coli complementation experiments

The complementation pattern of the derived episomes with the $E$. coli cys $C$ and $H$ mutants JM81 and JM96 was as predicted from the results of complementation tests in $S$. typhimurium. A mutant showing the complementation pattern of $S$. typhimurium cys $D$ was found among a group of mutants not previously characterised. The complementation pattern of strain JM73 (formerly designated $c y s P$ ) is identical to that of $S$. typhimurium cys 7 mutants, and that of strain JM57 to $S$. typhimurium cysI mutants.

(iv) Orientation of the cysCDHIJ genes in S. typhimurium

In $S$. typhimurium the order of the cysCDHIf genes is known (Demerec et al., 1963), as is the position of the group relative to the thy $A$ and $\arg B \operatorname{loci}$ (Sanderson, 1970) though its orientation was not known. In order to determine this a series of three- and four-point crosses was carried out. One $\mathrm{ml}$. of an overnight culture of the recipient strain was mixed with $1 \mathrm{ml}$. of a $\log$ phase culture of the donor strain and $1 \mathrm{ml}$. of nutrient broth. $0.2 \mathrm{ml}$. aliquots of the mixture were spread on 10 minimal agar plates supplemented with arginine and thymine and incubated for 44 hours. The colonies were replica plated on to minimal agar, minimal agar + arginine, minimal agar + 
thymine and minimal agar + arginine + thymine. The replica plates were incubated overnight and scored. The results of these experiments are given in table 4.

TABLE 4

Recombinants from the crosses to orient the cysCDHIJ gene group in $S$. typhimurium

\begin{tabular}{|c|c|c|c|c|}
\hline & Expt. 1 & Expt. 2 & Expt. 3 & Expt. 4 \\
\hline Donor & $\begin{array}{l}\mathrm{JM} 278 \\
\text { cys } C \\
\arg B\end{array}$ & $\begin{array}{l}\mathrm{JM} 278 \\
\text { cys } C \\
\operatorname{argB}\end{array}$ & $\begin{array}{l}0078 \\
\text { cys } C\end{array}$ & $\begin{array}{l}0078 \\
\text { cysC }\end{array}$ \\
\hline bacteria plated & $8.8 \times 10^{7}$ & $1.3 \times 10^{8}$ & $7.0 \times 10^{7}$ & $7.0 \times 10^{7}$ \\
\hline Recipient & $\begin{array}{l}\text { JM267 } \\
\text { cysf } \\
\text { thy }\end{array}$ & $\begin{array}{l}\text { JM267 } \\
\text { cysf } \\
\text { thy }\end{array}$ & $\begin{array}{l}\mathrm{JM} 279 \\
\text { cysf } \\
\text { argB }\end{array}$ & $\begin{array}{l}\text { JM280 } \\
\text { cysf } \\
\text { argB }\end{array}$ \\
\hline bacteria plated & $6.2 \times 10^{8}$ & $1.1 \times 10^{\circ}$ & $1.1 \times 10^{9}$ & $6.2 \times 10^{8}$ \\
\hline \multicolumn{5}{|l|}{ Recombinants } \\
\hline $\begin{array}{l}\text { Arg+ Thy+ } \\
\text { Arg' Thy- } \\
\text { Arg- Thy+ } \\
\text { Arg- Thy- }\end{array}$ & $\begin{array}{r}35 \\
150 \\
116 \\
17\end{array}$ & $\begin{array}{r}43 \\
162 \\
126 \\
9\end{array}$ & 194 & $\begin{array}{r}121 \\
18 \\
27 \\
144\end{array}$ \\
\hline $\begin{array}{l}\mathrm{Arg}^{+} \\
\mathrm{Arg}^{-}\end{array}$ & $\begin{array}{l}185 \\
131\end{array}$ & $\begin{array}{l}205 \\
135\end{array}$ & $\begin{array}{l}194 \\
372\end{array}$ & $\begin{array}{l}139 \\
171\end{array}$ \\
\hline$x(1)$ & $9 \cdot 15$ & $14 \cdot 4$ & 56 & $3 \cdot 3$ \\
\hline $\mathbf{P}$ & 0.001 & 0.001 & 0.001 & $0 \cdot 1-0 \cdot 05$ \\
\hline
\end{tabular}

Control plates showed less than one contaminant per plate.

Experiments 1 and 2 show a highly significant excess of $\mathrm{Arg}^{+}$clones among the progeny. Experiments 3 and 4 on the other hand show a significant excess of Arg- clones. The data from experiments 1, 2 and 4 were tested for heterogeneity; the contingency $\chi^{2}$ for 6 degrees of freedom is 6.89 , giving $0.5>\mathrm{P}>0.3$. Thus there are relatively as many $\mathrm{Arg}^{-}$recombinants in experiments 1 and 2 as there are Arg+ in experiment 4. The observations cannot therefore be due to preferential loss of $\mathrm{Arg}^{-}$recombinants. They are however consistent with the gene order (experiments 1 and 2):

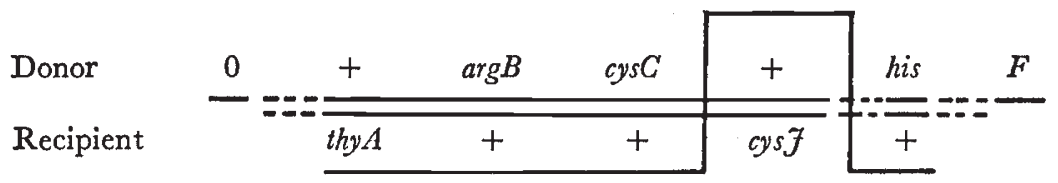

These experiments confirm the previously determined gene order, and show that the orientation of the cysCDHIJ gene cluster is not the same in $S$. typhimurium as in $E$. coli unless the result can be explained by exclusion of the Hfr arg allele from the recombinants.

\section{Commentary}

Provided that the episomal allele is dominant, the method here described for mapping $E$. coli genes has a number of advantages. It does not 
require the existence in $E$. coli of a mutant in the gene to be mapped: it is sufficient for the mutant to exist in an organism capable of harbouring and transmitting an $E$. coli episome. Since few clones need be scored, it is eminently suitable for the fine mapping of loci the phenotype of which is difficult to score. The high frequency of episome transfer also facilitates the mapping of unstable mutants. The use of a $S$. typhimurium strain (rather than an $E$. coli $\operatorname{rec} A$ strain) to carry the episome has the advantage that the chromosomal markers can more easily be manipulated. To have carried out the present experiments (which use markers close to $\operatorname{rec} A$ ) would have been much more arduous and time-consuming had an $E$. coli $\operatorname{rec} A$ strain been employed. Though there are advantages to be gained in using an episome carrying a fermentation marker this is in theory in no way necessary. The use of a positive selection technique for obtaining partially deleted episomes is convenient but presumably unnecessary. It should be pointed out that recessive resistance mutations which occur by change of protein specificity in haploids may also, in heterozygous diploids, occur by deletion of the dominant allele.

The result that the cysCDHIJ gene cluster is inverted in $S$. typhimurium, * though unexpected, is not surprising. Other examples are known, for instance that involving the tryptophan operon (Sanderson, 1970). It is consistent with the observation of Demerec and Ohta (1964) that $E$. coli $\times S$. typhimurium hybrids which have inherited one of these genes from $E$. coli have inherited them all, but have not inherited either of the flanking markers $\arg A$ or phe $A$, since two recombination events only one of which is within an inversion is likely to lead to an incomplete chromosome. Inversions of genetic material between species of Enterobacteriaceae are compatible with the current hypotheses of their evolution (Sanderson, 1971).

Acknowledgments.--I am grateful to many members of the department for helpful discussions and especially to Professor K. Mather and Dr M. J. Kearsey for advice on the use of statistics, to Messrs Burroughs Welcome and Co. for the gift of trimethoprim lactate, and to Professor S. Glover and Dr Claire Berg for supplying strains of $E$. coli.

\section{REFERENGES}

BERG, C. M., AND GURTISs, R. 1967. Transposition derivatives of an Hfr strain of Escherichia coli K12. Genetics, 56, 503-525.

DEMEREC, M., ADELBERG, E. A., CLARK, A. J., AND HARTMAN, P.E. 1966. A proposal for a uniform nomenclature in bacterial genetics. Genetics, 54, 61-76.

DEMEREC, M., Grllespie, D. H., AND MIzOBUCH, K. 1963. Genetic structure of the cysC region of the Salmonella genome. Genetics, 48, 997-1009.

DEMEREC, M., AND OHTA, N. 1964. Genetic analyses of Salmonella typhimurium $\times$ Escherichia coli hybrids. Proc. natn. Acad. Sci., U.S.A., 52, 317-323.

HAYES, w. 1957. The kinetics of the mating process in Escherichia coli. 7.gen. Microbiol., 16, 97-119.

ITIKAWA, H., AND DEMEREc, M. 1967. Ditto deletions in the cysC region of Salmonella typhimurium. Genetics, 55, 63-68.

JONES-MORTIMER, M. C. 1968. Positive control of sulphate reduction in Escherichia coli: isolation, characterisation and mapping of cysteineless mutants of $E$, coli K12. Biochem. 7., 110, 589-595.

MARSH, N. J., AND DUGGAN, D. E. 1972. Ordering of mutant sites in the isoleucine-valine genes of Escherichia coli by use of merogenotes derived from F14: a new procedure for fine-structure mapping. 7. Bact., 109, 730-740.

SANDERSON, K. E. 1970. Current linkage map of Salmonella typhimurium. Bact. Rev., 34, 176193.

* For S. typhimurium Salmonella geneticists please read E. coli. 
SANDERson, K. E. 1971. Genetics of the Enterobacteriaceae: Genetic homology in the Enterobacteriacae. Adv. Genet., 16, 35-51.

sMrTH, D. A. 1961. Some aspects of the genetics of methionine-less mutants of Salmonella typhimurium. F. gen. Microbiol., 24, 335-353.

sMrTH, D. A. 1971. S-amino acid metabolism and its regulation in Escherichia coli and Salmonella typhimurium. Adv. Genet., 16, 141-165.

STACEY, K. A., AND SIMSON, E. 1965. Improved method for the isolation of thymine-requiring mutants of Escherichia coli. 7. Bact., 90, 554-555.

SWANSTROM, M., AND ADAMS, M. H. 1951. Agar layer method for production of high titer phage stocks. Proc. Soc., exp. Biol. Med., 78, 372-375.

TAYLOR, A. L. 1970. Current linkage map of Escherichia coli. Bact. Rev., 34, 155-175. 
